\title{
Synchronisation par le nycthémère de l'activité cellulaire du noyau suprachiasmatique révélée par l'expression du protooncogène c-fos
}

Bien que l'intervention du noyau suprachiasmatique (NSC) dans le maintien de la périodicité de nombreux rythmes circadiens ait été largement démontrée, on ignore encore à l'heure actuelle les mécanismes cellulaires à l'origine de ce rôle synchronisateur. Cependant l'utilisation de marqueurs de l'activation neurale comme le protooncogène c-fos va peut-être permettre de lever un coin du voile.

Chez de nombreuses espèces de vertébrés, l'entraînement à la lumière repose sur des photorécepteurs oculaires et extra-oculaires. En ce qui concerne les mammiferes, il n'y a aucune preuve de l'intervention d'une photoréception extra-oculaire dans le contrôle des rythmes circadiens chez l'adulte, bien que la lumière puisse directement et transitoirement influencer l'activité de la glande pinéale chez le jeune [1], en particulier le taux de sérotonine. L'information photonique nécessaire à la genèse des rythmes circadiens chez les mammifères transiterait pour l'essentiel par le faisceau rétinohypothalamique et serait intégrée par le NSC [2]. Bien que le système circadien soit en général considéré comme un système d'oscillateurs multiples, le NSC est la seule structure dont le rôle de synchronisateur ait été réellement démontré. L'intégrité du NSC est nécessaire à l'entraînement des rythmes circadiens se manifestant au niveau du comportement des animaux nocturnes ou diurnes. La destruction du NSC conduit en effet à l'installation d'une arythmie [3, 4]. Le NSC n'est certainement pas le seul oscillateur à l'origine de l'ensemble des rythmes être le chef d'orchestre comme en atteste la restitution de la rythmicité de l'activité locomotrice par la transplantation de NSC fœtal chez des hamsters dont le NSC avait été préalablement lésé [5].

Le NSC - qui, comme son nom l'indique, est situé dans l'hypothalamus juste au-dessus du croisement des deux nerfs optiques - reçoit par ailleurs des informations photoniques par une voie indirecte via le noyau géniculé ventro-latéral en particulier sa portion dorsale, le feuillet intergéniculé [5]. Le NSC est composé de deux subdivisions principales différenciées par leur cytoarchitecture, par la morphologie et l'immunohistochimie de leurs populations neuronales et par la distribution des afférences visuelles. La subdivision ventrolatérale renferme des neurones exprimant le VIP (vasoactive intestinal peptide) et reçoit les afférences visuelles directes et indirectes. La subdivision dorsomédiane contient des neurones à vasopressine ou à somatostatine et ne reçoit aucun apport visuel. Par ailleurs, des cellules faiblement réactives à la GAD (glutamic acid decarboxylase) sont présentes dans tout le NSC de même qu'un riche réseau d'axones GAD positifs. Ces quatre types cellulaires définis chimiquement représentent une proportion importante de la population neuronale du NSC, mais très peu de ces neurones sont à l'origine des efférences du NSC. C'est ce qui a amené à considérer ces neurones comme pouvant constituer de nombreux oscillateurs individuels couplés en un important réseau de circuits locaux.

Nombreux sont les travaux ayant montré le caractère rythmique de l'activité électrique spontanée [6, 7] ou de l'activité métabolique [8] du NSC. Cette rythmicité du fonctionnement des cellules du NSC est endogène et spécifique au noyau comme l'ont récemment démontré les travaux de transplantations croisées sur deux souches de hamsters ayant chacune leur périodicité propre $(\mathrm{m} / \mathrm{s}$ $n^{\circ}$ 5, vol. 6, p. 488). Cependant, rien ne permettait de déterminer si cette rythmicité du fonctionnement du NSC résultait d'une propriété endogène des neurones ou de l'interaction complexe de l'ensemble des cellules constituant le noyau (interaction inter-neurones voire neurones-glie). Rusak et son équipe [9] viennent de démontrer que, une heure après le début d'une exposition à la lumière au cours de la scotophase (phase obscure), l'immunoréactivité pour Fos tout comme la production de l'ARN messager codant pour Fos étaient considérablement accrues dans la subdivision du NSC recevant les afférences visuelles (figure 1). Parallèlement, l'immunoréactivité à Fos est fortement augmentée dans le feuillet intergéniculé et le noyau géniculé latéro-ventral, alors qu'elle reste à un faible niveau de base dans le noyau géniculé latéro-dorsal qui est le relais primaire des voies visuelles. Cependant, cette augmentation de l'expression de $c$-fos ne coïncide pas avec le pic des décharges de l'activité spontanée ou le pic de la consommation de 2-désoxyglucose* qui apparaissent tous les deux au milieu de la phase lumineuse où il est impossible d'enregistrer une augmentation de l'expression de $c$-fos de façon spontanée ou

\footnotetext{
* Le 2-désoxyglucose est un analogue non métabolisé du glucose qui est un indicateur de l'activité métabolique des tissus.
} 


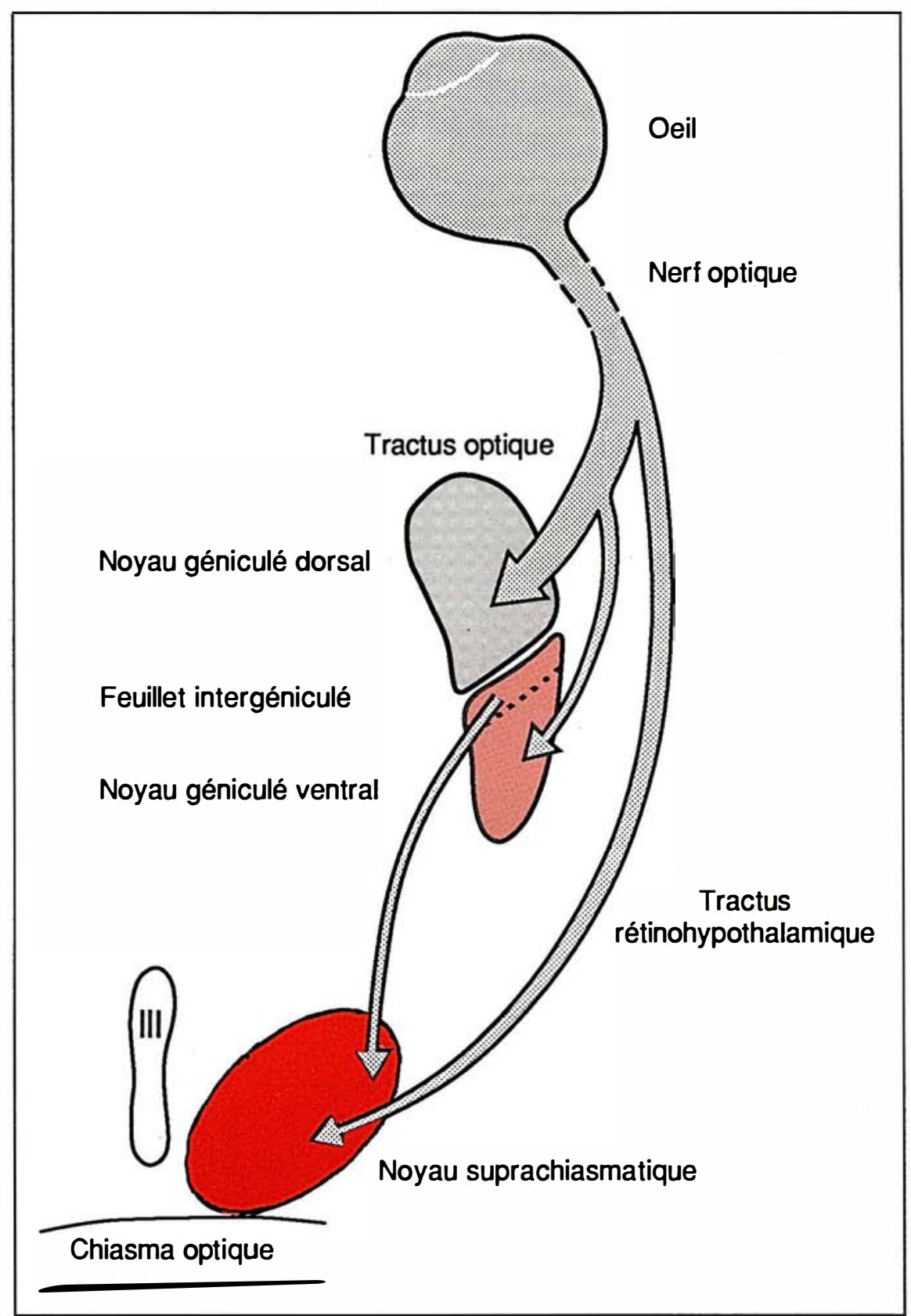

Figure 1. Les voies véhiculant l'information photonique nécessaire à la synchronisation des rythmes circadiens, depuis l'œil jusqu'à la subdivision ventro-latérale du noyau suprachiasmatique. III : $3^{e}$ ventricule. même déclenchée. L'expression de cfos varierait donc au cours du nycthémère en fonction des stimulations lumineuses, mais ne reflèterait pas forcément une augmentation de l'activité métabolique globale.

En attendant le détail des mécanismes moléculaires à l'origine des changements de phase du NSC, on va pouvoir rapidement savoir si toutes les populations cellulaires du NSC sont entraînées par les rythmes circadiens ou si seulement certaines d'entre elles sont concernées et lesquelles.

J. C.

1. Wetterberg L, Geller E, Yuwiller A. Harderian gland: an extraretinal photoreceptor influencing the pineal gland in neonatal rats ? Science 1970 ; 201 : 76-9.

2. Moore RY. Retinohypothalamic projections in mammals : a comparative study. Brain Res $1973 ; 49: 403-9$

3. Lehman ML, Silver R, Gladstone WR, Kahn RM, Gibson M, Bittman EL. Circadian rythmicity restored by neural transplant. Immunocytochemical characterization of the graft and its integration with the host brain. J Neurosci $1987 ; 7: 1626-38$.

4. Ralph MR, Foster RG, Davis FC, Menaker M. Transplanted suprachiasmatic nucleus determines circadian period. Science 1990 : 247: 975-8

5. Card JP, Moore RY. Ventral lateral geniculate nucleus efferents to the rat suprachiasmatic nucleus exhibit avian pancreatic polypeptide-like immunoreactivity. J Comp Neurol 1982 ; 206 : 390-6.

6. Inouye ST, Kawamura H. Persistence of circadian rythmicity in a mammalian hypothalamic "island " containing the suprachiasmatic nucleus. Proc Natl Acad Sci USA 1979 ; 76 : 5962-6.

7. Inouye ST, Kawamura H. Caracteristics of a circadian pacemaker in the suprachiasmatic nucleus. I Comp Physiol 1982 ; 146 : 153-60. 8. Schwartz WJ, Davidsen LC, Smith CB. In vivo metabolic activity of a putative circadian oscillator, the rat suprachiasmatic nucleus. $J$ Comp Neurol 1980 ; 189 : 157-67.

9. Rusak B, Robertson HA, Wisden W, Hunt SP. Light pulses that shift rythms induce gene expression in the suprachiasmatic nucleus. Science $1990 ; 248$ : 1237-40.

(n)

